\title{
Manejo quirúrgico de nariz mestiza con injerto de extensión septal
}

\author{
Deniss Calderón A. ${ }^{1}$, Doris Calderón A. ${ }^{2}$, \\ Mónica Tello L. ${ }^{2}$ y Ebingen Villavicencio C. ${ }^{2}$
}

\section{Surgical management of the mongrel nose with graft of septal extension}

Aim: The mongrel nose has very different characteristics from the caucasian one resulting in a surgical challenge for the facial cosmetic surgeon. The objective of the study was to describe the surgical management of the mongrel nose with graft of septal extension and to evaluate the aesthetic results. Materials and Method: Study of a series of cases of longitudinal section, sample of 25 patients. A surgical plan was performed using an open approach and the use of graft of septal extension, the pre and postoperative photos were compared by measuring the angle and projection of the nasal tip, together with the level of postoperative satisfaction of the patients. Descriptive statistics that included absolute and relative frequencies were used. Results: $56 \%$ belong to the female sex, $52 \%$ were placed spacer grafts, $8 \%$ needed an allotomy, $100 \%$ improved the nasolabial angle and only one patient presented septal hematoma at 10 days post surgery, it was resolved with drainage, the satisfaction survey of post-surgical patients showed a satisfactory result. Conclusions: The surgical technique with graft of septal extension is very useful, a correct definition, projection/rotation of the nasal tip and greater structural stability of the central tripod were achieved, with satisfactory harmonic results in its front and profile appearance, avoiding place grafts on the nasal tip.

Key words: rhinoplasty; graft of septal extensión; operative surgical procedures.

\section{Resumen}

Objetivo: La nariz mestiza presenta características muy diferentes de la caucásica resultando en un desafío quirúrgico para el cirujano estético facial. El objetivo del estudio fue describir el manejo quirúrgico de la nariz mestiza con injerto de extensión septal y evaluar los resultados estéticos. Materiales y Método: Estudio de una serie de casos de corte longitudinal, muestra de 25 pacientes. Se realizó un plan quirúrgico mediante abordaje abierto y el uso de injerto de extensión septal, se compararon las fotos pre y postoperatorias midiendo el ángulo y la proyección de la punta nasal, junto con el nivel de satisfacción postoperatoria de los pacientes. Se utilizó estadística descriptiva que incluyó frecuencias absolutas y relativas. Resultados: $56 \%$ de los pacientes corresponden al sexo femenino, al 52\% se colocó injertos espaciadores, $8 \%$ necesitó alotomía, el 100\% mejoró el ángulo nasolabial y solo un paciente presentó hematoma septal a los 10 días postquirúrgico, se resolvió con drenaje, la encuesta de satisfacción realizada a los pacientes postquirúrgicos mostró un resultado satisfactorio. Conclusiones: La técnica quirúrgica con injerto de extensión septal es de gran utilidad, se logró una correcta definición, proyección/rotación de la punta nasal y mayor estabilidad estructural del trípode central, con resultados armónicos satisfactorios en su apariencia de frente y de perfil, evitando colocar injertos en la punta nasal.

Palabras clave: rinoplastía; injerto extensión septal; procedimientos quirúrgicos operatorios.

\section{Introducción}

La nariz es la parte que sobresale en el rostro, como una elevación piramidal hueca que ocupa la parte central de la cara, cobra gran importancia en la simetría y balance estéticos que determinan la belleza facial; su modificación quirúrgica, rinoplastía, es una de las operaciones más demandadas y desafiantes; prueba las habilidades artísticas, el conocimiento de la anatomía, fisiología y el proceso de curación que debe tener el cirujano.

Dentro del campo de la cirugía plástica nasal,

\author{
'Universidad de Cuenca. \\ Ecuador. \\ ¿Universidad Católica de \\ Cuenca. Ecuador. \\ Recibido el 17 de julio \\ de 2019 y aceptado para \\ publicación el 27 de \\ noviembre de 2019.
}

Correspondencia a: Dra. Doris Eliana Calderón A. bq_elicalderon@yahoo.com 
Ortiz-Monasterio y colaboradores ${ }^{1}$, encontraron en pacientes de Latinoamérica la necesidad de obtener resultados de acuerdo a los cánones estéticos indoeuropeos. Según las características de las narinas y de la base nasal existe la clasificación que agrupa a las bases nasales en: leptorrina cuando el diámetro anteroposterior de la narina es mayor que el diámetro transversal, típica de los caucásicos; mesorrina, típica del mestizo y el oriental, en quienes ambos diámetros son casi iguales y presentan narinas redondas; y finalmente, la nariz de tipo platirrino, típica de la raza negra, el diámetro transversal es mayor que el anteroposterior ${ }^{2,3}$.

Los objetivos de la rinoplastía estética en el paciente mestizo son:

- Lograr una nariz más estrecha, prominente y con dorso elevado.

- Incrementar la proyección de la punta, estrechar la base alar, modificar el ángulo nasolabial.

- Producir un efecto general de angularidad que camufle el grosor de la piel.

De esta forma se emplean técnicas aumentativas de dorso y punta, combinadas con osteotomías y corrección de las alas nasales ${ }^{4,5}$.

La rinoplastía abierta fue un procedimiento ideado inicialmente para narices técnicamente difíciles, particularmente en casos con destrucción severa del

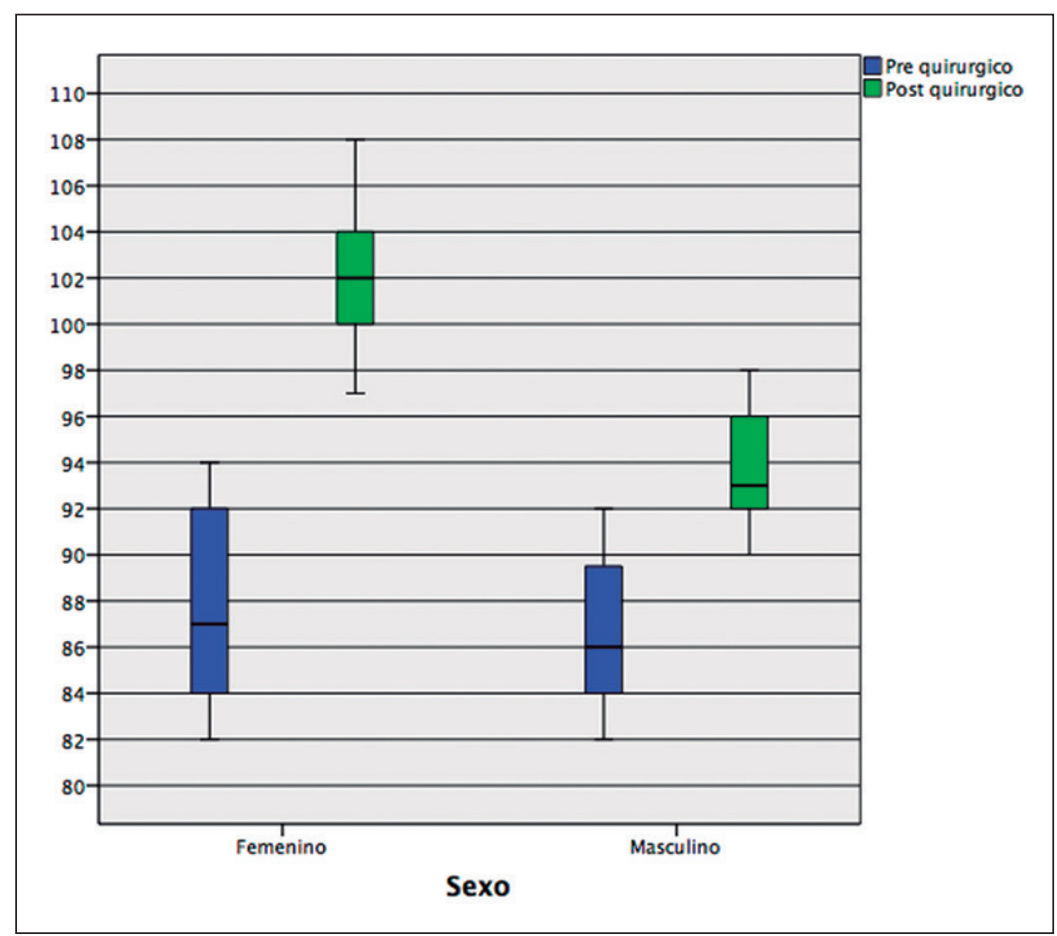

Figura 1. Ángulo nasolabial pre y postquirúrgico. armazón cartilaginoso secundario a traumatismos o por iatrogenia. No obstante, dada su utilidad, ha sido considerada como alternativa para rinoplastía estética primaria con resultados excelentes ${ }^{6}$. El abordaje abierto, aunque no está exento de complicaciones y controversias, ofrece un gran número de ventajas incuestionables en la actualidad ${ }^{7}$, facilita la realización de técnicas aumentativas de dorso, así como de refinamiento de la punta nasal con gran precisión. Otros autores ${ }^{8}$, han aplicado técnicas clásicas de reconstrucción nasal con la utilización de injertos espaciadores. Adham" utilizó otras opciones de injertos cartilaginosos para la reconstrucción nasal. En la nariz mestiza dicho abordaje permite al cirujano agotar al máximo todos los recursos técnicos posibles para obtener los cambios deseados ${ }^{10}$.

Por primera vez Byrd y colaboradores en 1997, lograron fijar un injerto de extensión septal en el tabique caudal o dorsal entre los dos cartílagos laterales inferiores mientras controlaba el alargamiento nasal y la proyección de la punta, la rotación y la forma ${ }^{11}$. Con la técnica de injerto de extensión septal se puede obtener un buen control de la proyección y rotación de la punta nasal, corrigiendo dichas anormalidades y mejorando la vista tanto de frente como de perfil ${ }^{12}$, con la ventaja adicional que los injertos de extensión septal cartilaginosos pueden ser adaptados y tallados ajustados a los requerimientos de cada caso en particular.

El objetivo del presente estudio fue describir el manejo quirúrgico de la nariz mestiza con injerto de extensión septal y evaluar los resultados estéticos.

\section{Material y Métodos}

Se trata de un estudio de una serie de casos, de corte longitudinal, con una muestra de 25 pacientes que cumplieron con los criterios de selección y fueron atendidos en Latino Clínica de la Ciudad de Cuenca-Ecuador, periodo 2017. Todos los pacientes presentaron consentimiento informado para la realización de la cirugía y la posterior difusión de los resultados. Se realizaron las mediciones del ángulo y la proyección de la punta nasal, se registraron los datos previos a la cirugía. La técnica quirúrgica incluyó:

- Asepsia y antisepsia con alcohol blanco y colocación de campos estériles.

- Infiltración nasal con solución de lidocaína al 1\% con adrenalina con una concentración 1:100.000.

- Abordaje abierto de rinoplastía con una incisión columelar de RETHI en "V" invertida e incisiones marginales bilateralmente. 
- Disección del sistema músculo aponeurótico superficial (SMAS) de la punta, a nivel de domos y dorso osteocartilaginoso.

- Resección del músculo depresor del septo nasal para promover la rotación de la punta y preparar la bolsa para un injerto de cartílago intercrural o de extensión septal.

- Disección subpericondrio del tabique nasal formando los túneles superiores e inferiores de cottle, para la recolección de injerto de cartílago septal, preservando la "L" puntal.

- Disección subperiosteal/subpericondrial del dorso con extensión lateral.

- Reducción más raspa de la giba osteocartilaginosa (cuando sea necesario).

- Colocación del injerto separadores (spreader graft) entre los cartílagos laterales superiores y el tabique para aumentar el ángulo de la válvula nasal interna. Realizar sólo cuando la reducción de la giba es necesaria o cuando el paciente tenía una disfunción de la válvula nasal preoperatoria.

- Recorte del borde cefálico de la crura lateral, dejando al menos $5 \mathrm{~mm}-7 \mathrm{~mm}$ y teniendo en cuenta que este es un cartílago débil.

- Formación de neodomos mediante sutura interdomal y/o transdomal.

- Colocación intercrural del injerto de extensión septal para proporcionar una buena plataforma a la punta nasal, produciendo rotación y proyección en caso necesario. El largo del injerto va desde la espina nasal hasta la proyección (longitud) que deseamos, se fija al septo con sutura no absorbible Nylon 5/0. La longitud y la angulación de esta estructura se determinan de acuerdo con las necesidades de cada caso (Figura 2).

- Se suturan las cruras medias al borde más caudal del injerto extensor (Figura 3).

- Osteotomías medias y laterales (depende de cada caso).

- Cierre meticuloso de la incisión columelar con Ethilon $6 / 0$

- Cierre de las incisiones mucosas Vicryl 5/0 (Figura 4).

- Valorar dimensión de base alar, por probable alotomías, en caso necesario.

- Colocación de micropore, más placa de yeso en dorso nasal.

Inmediatamente después de la cirugía se realizaron nuevas mediciones del ángulo y la proyección de la punta nasal, se registraron los datos. En el control postquirúgico realizado 4 semanas después, se aplicó una encuesta ${ }^{13}$ a los pacientes para valorar el grado de satisfacción en cuanto a resultados esté-

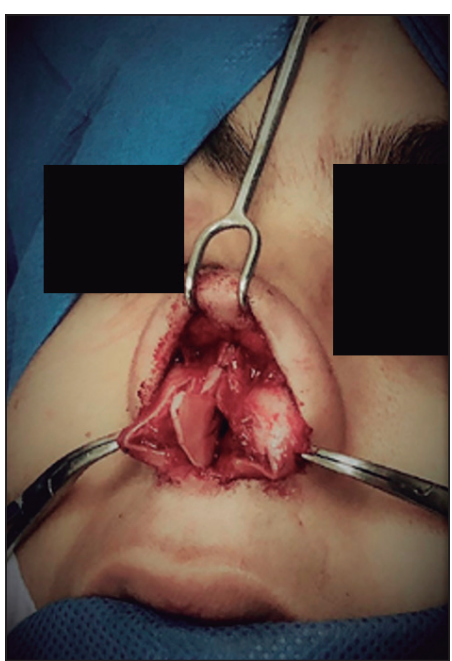

Figura 2. Colocación intercrural de injerto de extensión septal.

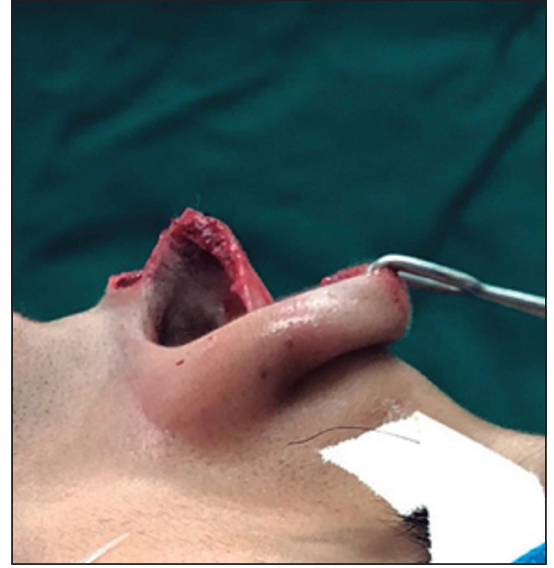

Figura 3. Unión de cruras medias a injerto extensión.

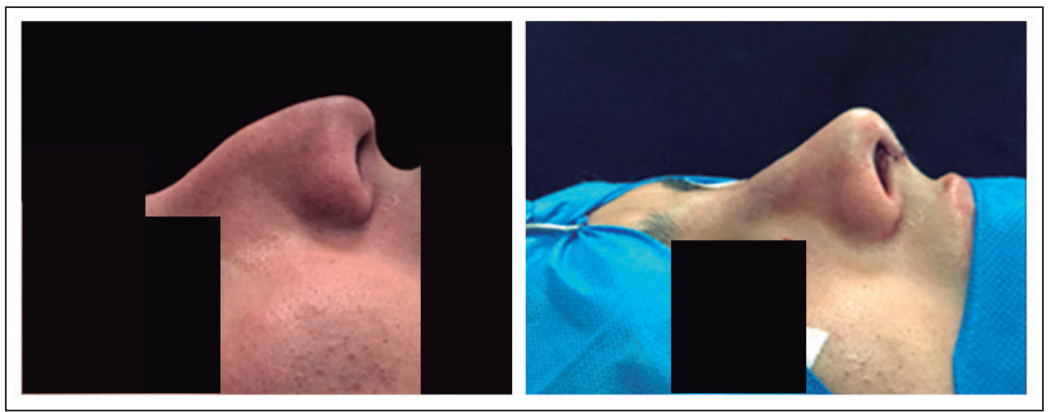

Figura 4. Vista lateral postquirúrgica inmediata.

ticos y funcionales. El instrumento utilizado se basó en la escala de Likert de cinco puntos, en donde el paciente expresa su acuerdo con la afirmación desde "completamente de acuerdo" hasta "completamente en desacuerdo".

\section{Resultados}

La evaluación de los resultados se realizó comparando las fotos preoperatorias y postoperatorias, se midió el ángulo nasolabial y el nivel de satisfacción postoperatoria de los pacientes. Se realizó el procedimiento quirúrgico de rinoseptoplastía funcional con abordaje abierto más colocación de injerto de extensión septal a 25 pacientes que cumplieron con los criterios de selección y acudieron al centro Latino Clínica de la ciudad de Cuenca-Ecuador, de los cuales el $56 \%$ pertenecen al sexo femenino 
Tabla 1. Distribución de la población según sexo

\begin{tabular}{|lcc|}
\hline & Frecuencia & Porcentaje \\
Femenino & 14 & 56 \\
Masculino & 11 & 44 \\
Total & 25 & 100 \\
\hline
\end{tabular}

Tabla 2. Distribución de la población según edad

\begin{tabular}{|lrc|}
\hline & Frecuencia & Porcentaje \\
\hline Menos de 20 & 12 & 48 \\
De 20 a 30 & 9 & 36 \\
De 30 a 40 & 4 & 16 \\
Total & 25 & 100 \\
\hline
\end{tabular}

Tabla 3. Injerto espaciadores colocados

\begin{tabular}{|lcc|}
\hline & Frecuencia & Porcentaje \\
\hline No & 12 & 48 \\
Sí & 13 & 52 \\
Total & 25 & 100 \\
\hline
\end{tabular}

Tabla 4. Prevalencia de alotomías

\begin{tabular}{|lcc|}
\hline & Frecuencia & Porcentaje \\
No & 23 & 92 \\
Sí & 2 & 8 \\
Total & 25 & 100 \\
\hline
\end{tabular}

Tabla 5 Prevalencia de complicaciones

\begin{tabular}{|lcc|}
\hline & Frecuencia & Porcentaje \\
No & 24 & 96 \\
Sí & 1 & 4 \\
Total & 25 & 100 \\
\hline
\end{tabular}

Tabla 6. Diferencia de ángulo de acuerdo a sexo

\begin{tabular}{|lccr|}
\hline & \multicolumn{2}{c}{ Sexo } & \\
Media & Femenino & Masculino & Total \\
Desviación estándar & 14,21 & 7,27 & 11,16 \\
Mínimo & 3,79 & 2,49 & 4,77 \\
Máximo & 8,00 & 2,00 & 2,00 \\
\hline
\end{tabular}

(Tabla 1), 48\% pacientes menores de 20 años (Tabla 2), al 52\% se colocó injerto espaciadores (Tabla 3 ), un $8 \%$ necesitó alotomía (Tabla 4); en el 100\% de pacientes mejoró el ángulo nasolabial se tuvo como promedio 11,16 (d.s. 4,77) grados de diferencia entre el inicio y el final, sin embargo, esta diferencia fue mayor en las pacientes de sexo femenino 14,21 (d.s.3,79) frente a un 7,27 (d.s.2,49) en el grupo de varones (Figura 1), solo un paciente presentó una complicación postquirúrgica (Tabla 5) hematoma septal a los 10 días, que fue resuelto con drenaje inmediato. Posteriormente, se aplicaron encuestas a todos los pacientes postquirúrgicos, la información recopilada señaló, en términos generales, que el procedimiento fue evaluado satisfactoriamente. Presentamos 2 casos de pacientes sometidos al procedimiento:

- Caso 1. Paciente femenina de 24 años de edad, con una punta nasal ancha, piel gruesa y cartílagos alares débiles, con falta de proyección, los resultados postoperatorios mostraron una adecuada mejoría en la definición de la punta nasal y estrechamiento, con una proyección y rotación adecuada (Figura 5).

- Caso 2. Paciente femenina de 17 años de edad, con giba dorsal, columela corta, ángulo naso labial agudo y laterorrinea, en la vista postquirúrgica se evidencia dorso recto, con una adecuada proporción punta-columelar, mejorando el ángulo nasolabial, mejoría en su rotación y proyección de la punta nasal (Figura 6).

\section{Discusión}

Pueden ser numerosas las causas de deformidades de la nariz, que afectan principalmente a la punta nasal y al dorso. En Sudamérica predominan los pacientes de raza mestiza que presentan nariz con dorso convexo, base nasal ancha y punta nasal desproyectada, que es provocada porque los cartílagos alares son más delgados y débiles, un tabique nasal corto, brindando un soporte estructural escaso lo que lleva a presentar un ángulo nasolabial agudo, características típicas que fueron observadas en los pacientes que participaron en este estudio. Los pacientes que presentan este tipo de deformidades nasales tienen el trípode nasal cartilaginoso (formado por las cruras medias, intermedias y laterales) muy débil, lo que genera un sostén de la punta nasal muy limitado ${ }^{14}$ como se evidenció en los pacientes sometidos a cirugía en esta investigación. El injerto de extensión septal que representa una técnica quirúrgica que amerita un análisis preoperatorio exhaustivo permite 
reforzar el pilar central, provocando una adecuada proyección-rotación y, además, adecuada definición de la punta nasal, con un correcto ángulo nasolabial, la estabilidad del injerto es importante para mejores resultados a largo plazo y complicaciones mínimas o recaídas, que mejora la vista tanto de frente como la del perfil ${ }^{14,15}$, en nuestra investigación la colocación del injerto cumplió con lo mencionado, tal como lo señalan Fernando Krljih, Graft Myung y Hoon Kim en sus investigaciones. Al realizar los nuevos domos (neodomos) que son reubicados gracias al injerto de extensión septal, podemos lograr una punta nasal bien definida cambiando el aspecto de la punta nasal bullosa y logrando estándares de belleza nasal, sin necesidad de injertos en punta nasal ${ }^{16}$. En comparación con el puntal columelar en pacientes con cartílago lateral inferior o bóveda media débil, se ha encontrado que el injerto de extensión septal es más favorable para mantener la proyección de la punta a través de un soporte más fuerte ${ }^{11}$, así lo podemos evidenciar en nuestra investigación. En el posoperatorio la proyección nasal se incrementó, al igual que la longitud nasal, mejorando el ángulo naso - labial. La cicatriz columelar que se produce al realizar el abordaje abierto, fue de buena calidad, bien tolerada y con el paso del tiempo prácticamente imperceptible en todos nuestros pacientes, ya que ninguno mostró inconformidad al respecto, con lo cual coincidimos con los resultados de otros autores $^{17,18}$.

\section{Conclusiones}

Las técnicas de rinoplastía actuales y en especial las de abordaje abierto, nos permiten producir cambios nasales con mejoría estética para los pacientes mestizos. La implementación del injerto de extensión septal es de gran utilidad, logrando una correcta definición, proyección/rotación de la punta nasal y mayor estabilidad estructural del trípode central.

En la presente investigación se obtuvieron resultados armónicos satisfactorios con una adecuada definición de la punta nasal, así como una mejoría en la proporción punta-columelar, mejorando su apariencia tanto de frente como de perfil y evitando colocar injertos en la punta nasal.

\section{Agradecimientos}

A Latino Clínica, por facilitarnos las instalaciones, laboratorios y medios que nos permitieron llevar a cabo la investigación.

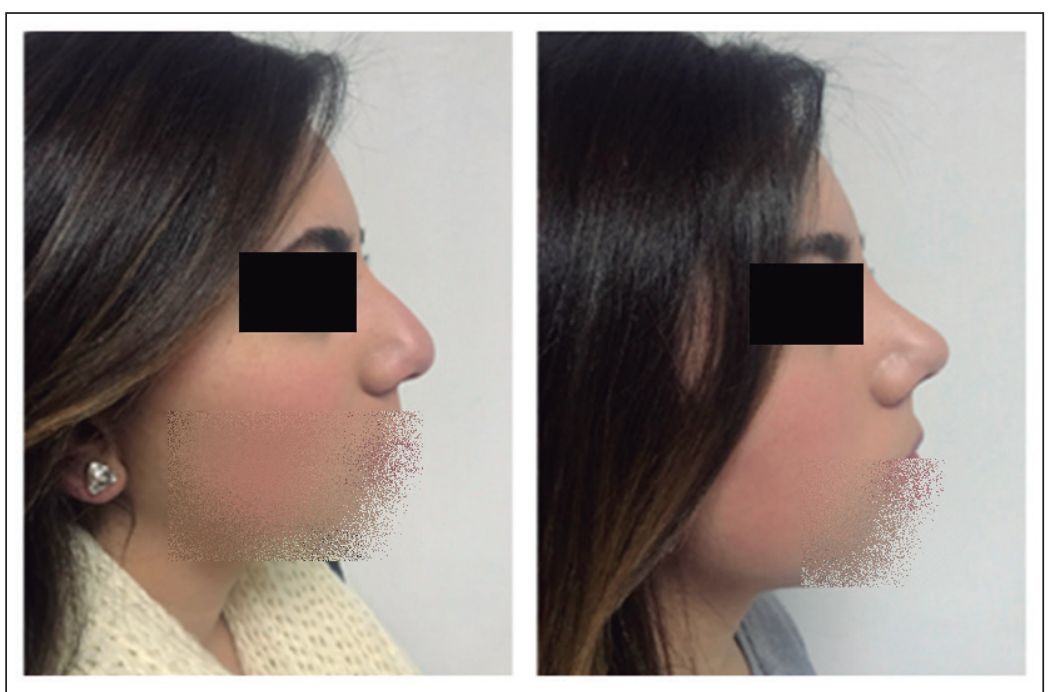

Figura 5. Vista lateral pre y postquirúrgica caso clínico 1. Paciente femenina de 24 años de edad, quien consulta por necesidad de mejorar algunos aspectos estéticos nasales, dificultad respiratoria. Imágenes pre y postquirúrgicas de 3 meses de evolución. Se evidencia el ángulo naso septal: $94^{\circ}$ preqx, $102^{\circ}$ postqx

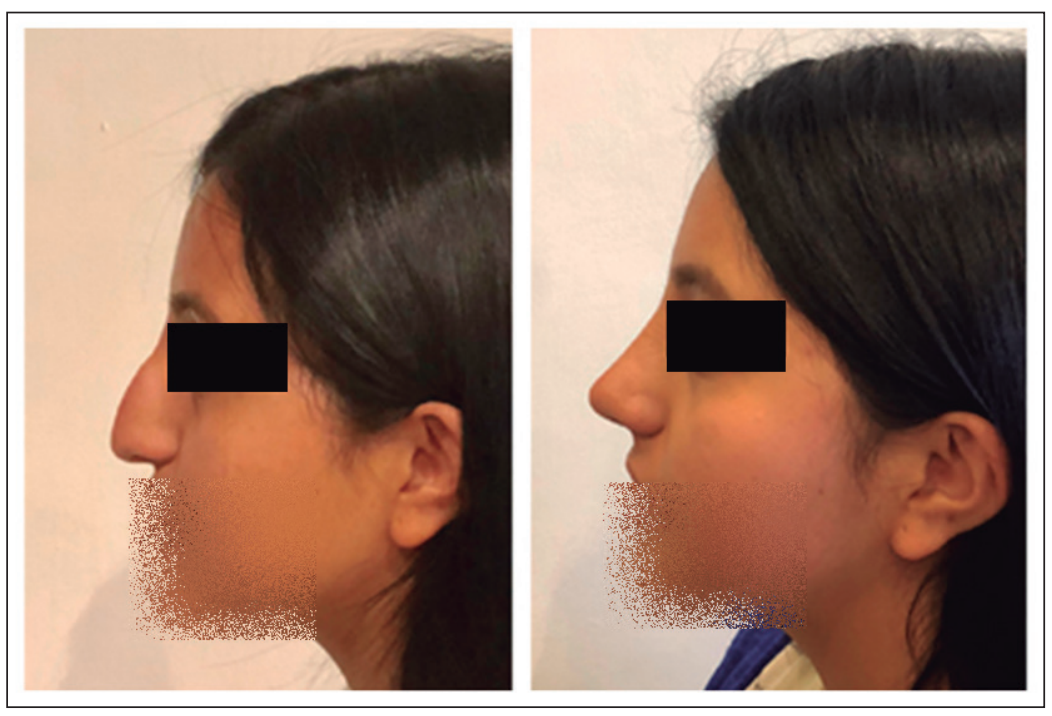

Figura 6. Vista lateral pre y postquirúrgica. Caso 2. Paciente femenina de 17 años de edad, acude a control por dificultad respiratoria derecha, mejorar estética. En las imágenes del postoperatorio (3 meses) se aprecia mejoría en la definición de la punta nasal y estrechamiento, con una proyección y rotación adecuada, ausencia de giba osteocartilaginosa. Se evidencia la medición del ángulo naso septal: $85^{\circ}$ preqx y $108^{\circ}$ postqx.

\section{Responsabilidades éticas}

Protección de personas y animales. Los autores declaran que para esta investigación no se han realizado experimentos en seres humanos ni en animales. 
Confidencialidad de los datos. Los autores declaran que en este artículo no aparecen datos de pacientes.

Conflictos de interés: no hay.
Fuentes de financiación: La presente investigación no ha recibido ayudas específicas provenientes de agencias del sector público, sector comercial o entidades sin ánimo de lucro.

\section{Bibliografía}

1. Ortiz-Monasterio F, Olmedo A. Rhinoplasty on the mestizo nose. Clin Plast Surg. 1997;4:89.

2. McCarthy JG, Word-Smith D. Rinoplastía. Cirugía Plástica La Cara II. Buenos Aires: Ed. Médica Panamericana S.A.; 1994.

3. Quiroz GF. Anatomía humana. Editorial Porrúa. México. 1991 63:93.

4. Ortiz Monasterio F, Olmedo A, Ortiz L. The use of cartilage grafts in primary rhinoplasty. Plast Reconstr Surg. 1981;67:597-605

5. Gruber R. Rhynoplasty state of the art. St Louis: Mosby; 1993. 75:83.

6. Johnson CM, Toriumi DM. Open Structure Rhinoplasty: The Concept. In: Johnson CM, Toriumi DM: Open Structure Rhinoplasty. W.S. Saunders Co, Philadelphia, PA, USA, 1990.

7. Aiach G. Atlas de rinoplastía y de la vía de abordaje externo. Vía de abordaje externo transcolumelar. Barcelona: Masson; 1992.13:40.

8. Rohrich RJ, Hollier LH. Use of spreader grafts in the external approach to rhinoplasty. Clin Plast Surg. 1996; 23 : 255-62.

9. Adham MN. A new technique for nasal tip cartilage graft in primary rhinoplasty. Plast Reconstr Surg 1996; 97:649-55.

10. Gálvez J, Millán I. Cambios antropométricos en la nariz mestiza después de rinoplastía abierta" Rev Cubana Cir. 2007;46-52.

11. Byrd HS, Andochick S, Copit S, Walton KG. Septal extension grafts: a method of controlling tip projection shape. Plast Reconstr Surg 1997;100:999-1010.

12. Toriumi DM, Patel AB, DeRosa J. Correcting the short nose in revision rhinoplasty. Facial Plast Clin North Am. 2006; 14:343-55

13. Danilla S, Ríos M, Cuevas P, Calderón
ME, Domínguez C, Di Silvestre P, et al. Medición de los resultados estéticos y funcionales desde la perspectiva del paciente en rinoplastía: creación del instrumento NOSE-QoL. Rev Chil Cir. 2014;66:38-44.

14. Krljih F. Injerto de extensión septal, Revista Faso 2013;20:18-22.

15. Graft Myung - Hoon Kim. An Introduction to the Septal Extension. Arch Plast Surg. 2014;41:2934.

16. Kang JG, Ryu J. Nasal tip surgery using a modified septal extension graft by means of extended margina incision. Plast Reconstr Surg. 2009; 123:343-52.

17. Anderson JR, Johnson JR, Adamson P. Open rhinoplasty. An assessment. Otolaryngol Head Neck Surg. 1982;90:272-74.

18. Gunter JP. The Merits of the Open Approach in Rhinoplasty. Plast Reconstruc Surg. 1997;99:863-7. 